ARTICLE

https://doi.org/10.1038/s41467-019-14238-3

\title{
The molecular basis of pyrazinamide activity on Mycobacterium tuberculosis PanD
}

\author{
Qingan Sun (10 ${ }^{1}$, Xiaojun $\mathrm{Li}^{1}$, Lisa M. Perez (1) ${ }^{2}$, Wanliang Shi ${ }^{3}$, Ying Zhang ${ }^{3} \&$ James C. Sacchettini ${ }^{1 \star}$
}

Pyrazinamide has been a mainstay in the multidrug regimens used to treat tuberculosis. It is active against the persistent, non-replicating mycobacteria responsible for the protracted therapy required to cure tuberculosis. Pyrazinamide is a pro-drug that is converted into pyrazinoic acid (POA) by pyrazinamidase, however, the exact target of the drug has been difficult to determine. Here we show the enzyme PanD binds POA in its active site in a manner consistent with competitive inhibition. The active site is not directly accessible to the inhibitor, suggesting the protein must undergo a conformational change to bind the inhibitor. This is consistent with the slow binding kinetics we determined for POA. Drug-resistant mutations cluster near loops that lay on top of the active site. These resistant mutants show reduced affinity and residence time of POA consistent with a model where resistance occurs by destabilizing the closed conformation of the active site.

\footnotetext{
${ }^{1}$ Department of Biochemistry and Biophysics, Texas A\&M University, College Station, TX, USA. ${ }^{2}$ Laboratory for Molecular Simulation, Texas A\&M University, College Station, TX, USA. ${ }^{3}$ Department of Molecular Microbiology and Immunology, Bloomberg School of Public Health, Johns Hopkins University, Baltimore, MD 21205, USA. *email: sacchett@tamu.edu
} 
n 2018, tuberculosis (TB) claimed more lives than any other infectious disease ${ }^{1}$. This is primarily because $\mathrm{TB}$ is rampant precisely in the areas where it is most challenging to treat due to the poverty of those areas and the lengthy treatment course. Adding to the difficulty in effective treatment is widespread drug resistance. Of the four front-line anti-TB drugs (isoniazid, rifampin, pyrazinamide (PZA), and ethambutol), PZA has been an irreplaceable component in clinical $\mathrm{TB}$ regimens due to its unique activity against persisters in chronic $M t b$ infections and ability to shorten treatment times ${ }^{2}$. It is for these reasons that PZA is recommended by the WHO as an integral component in the treatment of multidrug resistant $\mathrm{TB}^{3}$.

Despite the clinical importance of PZA and the early recognition of its anti-TB activity back in the 1950s, its mechanism of action has not been fully understood ${ }^{2,4}$. This is primarily because in vitro mode-of-action experiments are complicated by the fact that PZA is not active against $M t b$ cultures grown in normal media ${ }^{5}$. It is active under mildly acidic $\mathrm{pH}$, but even then it is not very potent with minimum inhibitory concentrations between $0.4-1.6 \mathrm{mM}^{6,7}$. What is certain is that PZA is a prodrug that is converted into pyrazinoic acid (POA) by the enzyme pyrazinamidase, PncA $(\mathrm{Rv} 2043 \mathrm{c})^{8}$, and that most of the PZA clinical resistance arises from loss of function mutations in pncA. However, multiple mechanisms of action and targets have been reported for $\mathrm{PZA}^{9-18}$. Recently, Zhang et al. reported several PZA resistant mutations that mapped to the $M t b$ panD gene ${ }^{19,20}$, suggesting it was a target of PZA. PanD is an aspartate decarboxylase responsible for the formation of $\beta$-alanine from L-aspartate, which is part of the pantothenate biosynthetic pathway, essential for vitamin B5 and coenzyme A biosynthesis in $M t b^{21}$.

Here, we present biochemical and structural evidence that POA inhibits $M t b$ PanD in a competitive fashion and that it binds with a high degree of complementarity to the active site of the enzyme. The binding affinity is consistent with the potency of PZA against $M t b$. In addition, the majority of the PZA drug-resistant mutations are located close to the catalytic center interacting with amino acids on two loops that enclose the active site. These results serve to solidify the prior clinical sequencing data that implicated PanD as the target for PZA.

\section{Results}

POA is a competitive inhibitor of PanD. Using purified recombinant $\mathrm{PanD}$, we have characterized the inhibition of the enzyme by POA with a coupled enzyme assay. The data clearly showed that POA inhibition is competitive with aspartate (Fig. 1a) in contrast to previously published results, placing it distant from the active site 22 . POA has a $K_{\mathrm{i}}$ of $0.78(0.05) \mathrm{mM}$, which is comparable to the $K_{\mathrm{M}}$ of $1.08(0.06) \mathrm{mM}$ for the substrate aspartate. We also tested PZA, nicotinic acid (NA), and two POA analogs with chlorine atom at the 5-(para) or 6-(meta) position (Fig. 1b). None of these compounds inhibited $M t b$ PanD at concentrations up to $20 \mathrm{mM}$, except 6-Cl-POA which was also competitive and slightly less potent compared to POA $\left(K_{\mathrm{i}}=1.00\right.$ (0.04) mM, Fig. 1c).

We next used isothermal titration calorimetry (ITC) to directly measure POA's binding affinity. POA, as a weak acid, can generate robust ITC signals on its own due to $\mathrm{pH}$-dependent proton dissociation ${ }^{12}$. We also observed that $M t b$ PanD is sensitive to acidic $\mathrm{pH}$, and it begins to precipitate $<\mathrm{pH}$ 7.0. To prevent $\mathrm{pH}$ related artifacts in the ITC, we buffered both the POA and the protein in $100 \mathrm{mM}$ Tris buffer at $\mathrm{pH} 7.5$ (Fig. 1d and Supplementary Fig. 1). The ITC data fit to a single-site model, with a $K_{\mathrm{d}}$ for POA of $0.71(0.03) \mathrm{mM}(n=5)$ and that of $6-\mathrm{Cl}-$ POA as $1.09(0.04) \mathrm{mM}(n=3)$. These values are in very good agreement with the $K_{\mathrm{i}}$ values of $0.78 \mathrm{mM}$ and $1.0 \mathrm{mM}$ described above. We saw no binding of NA, indicating that the single-atom change on the pyrazine ring $(\mathrm{N} 1->\mathrm{C})$ abolished the interaction between POA and $M t b$ PanD. The ITC results for both POA and 6-Cl-POA, showed that the measured $\Delta H$ was negative for both inhibitors $\left(-4200(200) \mathrm{cal} \mathrm{mol}^{-1}\right.$ and $-3300(100) \mathrm{cal} \mathrm{mol}^{-1}$ ), while the $\Delta S$ was relatively small $\left(0.1(0.7) \mathrm{cal} \mathrm{mol}^{-1} \mathrm{deg}^{-1}\right.$ and $\left.2.2(0.5) \mathrm{cal} \mathrm{mol}^{-1} \mathrm{deg}^{-1}\right)$. This indicated that the binding was enthalpy driven and that the POA and 6-Cl-POA interactions with the enzyme were primarily electrostatic or hydrogen bonds. This is in fact what we observed in the structure: hydrogen bonds and electrostatic interactions are critical for binding of POA to PanD, as described below. It is difficult to interpret the ITC results from a previous publication where POA was reported to have a $K_{\mathrm{d}}$ of $6.1 \mu \mathrm{M}$ for $M t b \mathrm{PanD}$, which was based on ITC in unbuffered water ${ }^{22}$. The $\mathrm{pH}$ of the POA in unbuffered water is $<3.0$ which brings into question the physiological relevance of the result.

To confirm our ITC binding data and to determine the on-rate and off-rate of binding, we employed an orthogonal method, BioLayer interferometry $(\mathrm{BLI})^{23}$, to measure POA binding to PanD. We monitored the real-time association and dissociation between POA, and $M t b$ PanD on the surface of the biosensor. The $k_{\text {on }}$ rate for POA is $3.5(0.6) \mathrm{M}^{-1} \mathrm{~s}^{-1}$, and the $k_{\mathrm{off}}$ is $0.0027(0.0001) \mathrm{s}^{-1}$. The $K_{\mathrm{d}}$ was derived from $k_{\text {off }} / k_{\text {on }}$ as $0.8(0.1)$ $\mathrm{mM}$ and matched well with the affinity we measured through ITC (Supplementary Fig. 2). The relatively high $K_{\mathrm{d}}$ is primarily related to the slow on-rate of POA. In short, POA binds $M t b$ PanD with a slow on-rate, but POA-PanD complex is very tight once formed, as evidenced by the very slow off-rate. The observed kinetics of the POA-PanD interaction may help to explain the paradox of PZA as a slow-acting, low-potency, but highly effective TB drug.

POA binds to the active site of PanD. Crystals of $M t b$ PanD were produced using the hanging-drop vapor diffusion method. The crystal structures of apo $M t b$ PanD and the $M t b$ PanD: POA complex were solved using molecular replacement and both structures were refined to $2.7 \AA$ resolution (Supplementary Note 1). The statistics describing the quality of the diffraction data and structures are in Supplementary Table 1. The first 115 residues were clearly visible in the electron density map, while the last 24 residues could not be built due to the flexibility at the C-terminus. PanD is a proenzyme and in the structure, we observed the processed active enzyme after cleavage between Gly24 and Ser25, and modification of Ser25 to a pyruvoyl group (Pyr25). The active protein consists of a $\beta$ chain (Met1-Gly24) and an $\alpha$ chain (Pyr25 to ILE115). In the POA complex structure, there was one molecule of POA bound to each $M t b$ PanD active site, which was located at the interface between two adjacent subunits of the PanD tetramer (Fig. 2a). The density for POA was clearly visible in a difference electron density map made by subtracting the structure factor amplitudes of the apo crystal diffraction data from that of the PanD:POA complex, as well as a polder OMIT map ${ }^{24}$ (Fig. 2b, c). The superimposition of the PanD:POA structure with the crystal structure of Helicobacter pylori PanD bound to a substrate analog, isoasparagine (pdb: $1 \mathrm{UHE}^{25}$ ), showed that there was good agreement in the hydrogen bonding of the inhibitor and substrate analog to the protein. Specifically, an oxygen from the carboxylate of POA is positioned very close to an oxygen of the isoasparagine $\gamma$-carboxyl group and forms hydrogen bonding interactions with the guanidinium group of Arg54* (Supplementary Fig. 3) ${ }^{25}$.

Because of the internal symmetry of the POA there are two ways to fit POA to the electron density. We choose to position the molecule based on optimal hydrogen bonding (Fig. 2b) 
a

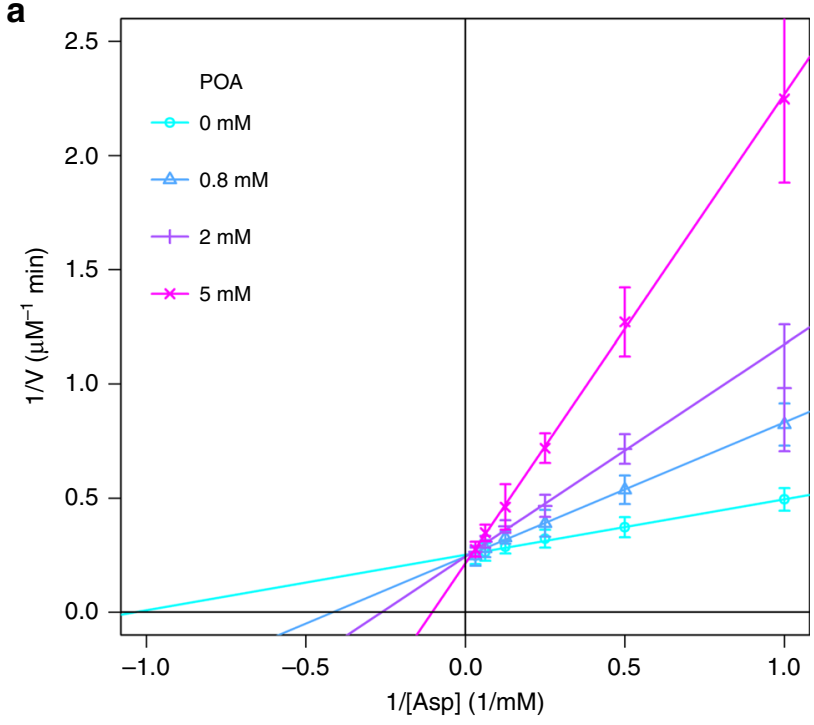

b

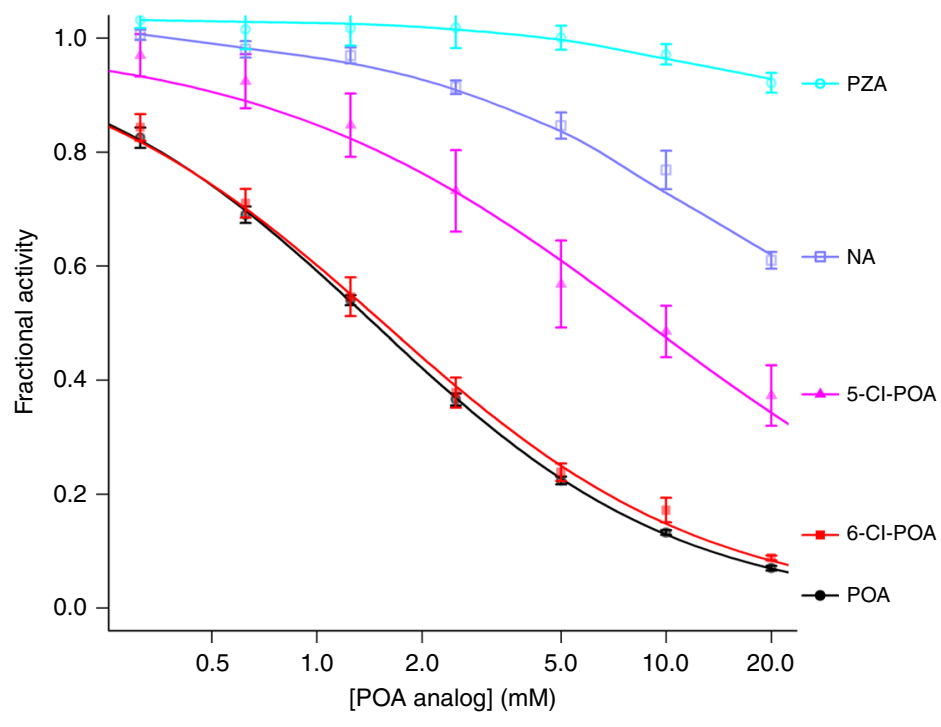

c

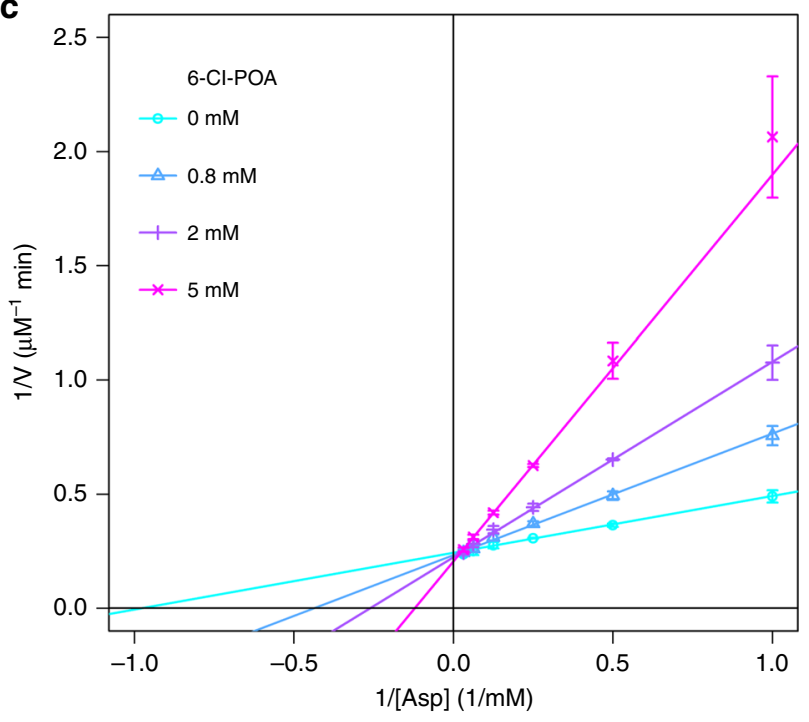

d<smiles>NC(=O)c1cnccn1</smiles><smiles>O=C(O)c1cccnc1</smiles><smiles>O=C(O)c1cnc(Cl)cn1</smiles>

Time $(\min )$

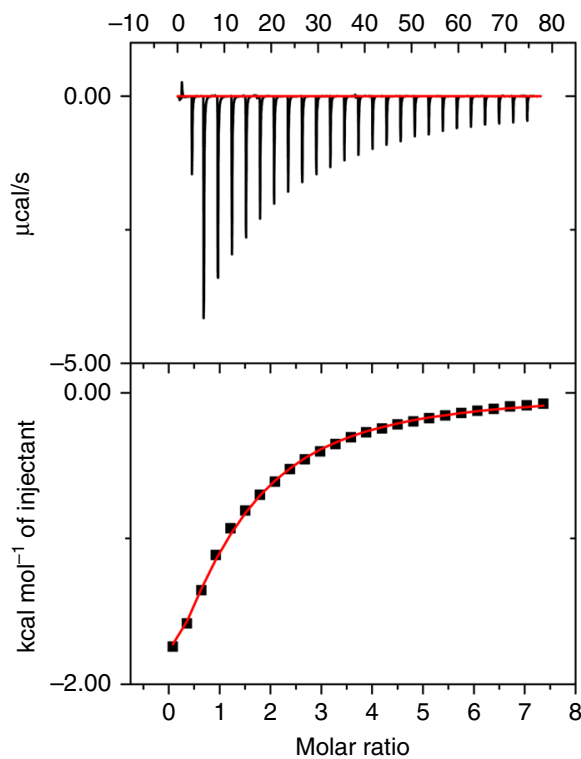

Fig. 1 Biochemical characterization of the interaction between Mtb PanD and POA. a POA showed competitive inhibition of Mtb PanD. Lineweaver-Burk plots of $M t b$ PanD activity in the presence of various concentrations of POA prepared in triplicate. The data were fitted with a competitive inhibition model, yielding $K_{\mathrm{i}}=0.78(0.05) \mathrm{mM}, K_{\mathrm{M}}=1.08(0.06) \mathrm{mM}$, and $k_{\text {cat }}=0.330(0.006) \mathrm{s}^{-1}$. b Inhibition of PanD activity with POA analogs. Dose-response curves were measured using the described PanD assay with pyrazinamide (PZA), nicotinic acid (NA), 5-Cl-POA, 6-Cl-POA, and POA. There were six replicates. c 6-Cl-POA inhibits Mtb PanD comparatively to POA. The plot was indicative of a competitive model of inhibition with $K_{\mathrm{i}}=1.00(0.04) \mathrm{mM}, K_{\mathrm{M}}=1.12(0.04$ $\mathrm{mM})$, and $k_{\mathrm{cat}}=0.350(0.003) \mathrm{s}^{-1}$. d Isotherm calorimetry of Mtb PanD with POA. The top panel shows the heat released per injection of inhibitor, as $\mu c a l$ $\mathrm{s}^{-1}$; while the bottom panel shows the change in enthalpy $\left(\mathrm{kcal} \mathrm{mole}^{-1}\right)$ as a function of the molar ratio of POA to PanD. Titrations were performed at $20^{\circ}$ $\mathrm{C}$ using $100 \mathrm{mM}$ Tris buffer ( $\mathrm{pH} \mathrm{7.5)} \mathrm{for} \mathrm{both} \mathrm{the} \mathrm{protein} \mathrm{solution} \mathrm{and} \mathrm{POA} \mathrm{titrant.} \mathrm{The} \mathrm{data} \mathrm{were} \mathrm{fitted} \mathrm{with} \mathrm{a} \mathrm{single-site} \mathrm{binding} \mathrm{model.} \mathrm{From} \mathrm{five} \mathrm{separate}$ experiments, it was calculated that $K_{d}=0.71(0.03) \mathrm{mM}, \Delta H=-4200(200) \mathrm{cal} \mathrm{mol}^{-1}, \Delta S=0.1(0.7) \mathrm{cal} \mathrm{mol}^{-1} \mathrm{deg}^{-1}$. Error bars were defined as standard deviations. Source data are provided as a Source Data file.

This fit was confirmed when we docked POA into this binding pocket with Glide 26 . The prediction put POA in the same pose as in the crystal structure with all the interactions preserved, the root-mean-square difference on non-hydrogen atoms of POA was only $0.29 \AA$. The molecular surface of the active site indicated there was room to add a substituent to the pyrazine ring of POA at the 6-position based on the pose we selected. This was consistent with the finding that $6-\mathrm{Cl} \mathrm{POA}$ was an inhibitor of $\mathrm{PanD}$ and 5-Cl $\mathrm{POA}$ showed no inhibition. We cocrystallized Mtb PanD with the 6-Cl POA analog and refined the structure to $2.25 \AA$ (Supplementary Table 1).
A polder OMIT map showed clear electron density for the 6$\mathrm{Cl}$ and it was oriented with the pyrazine ring in the same orientation as we fit POA (Fig. 2d, e).

The POA binding pocket is relatively small $\left(206 \AA^{3}\right)$ and not easily accessible, as there is only a narrow tunnel leading from the active site to the inside barrel of the PanD tetramer. With a diameter of $\sim 3.4 \AA$, neither the substrate nor inhibitor could pass through the tunnel. The chlorine atom of 6-Cl-POA resides in this tunnel. There is also a small protrusion of space on the opposite side of the tunnel that extends toward the outside surface of the tetramer, but it is blocked from being open to 
a

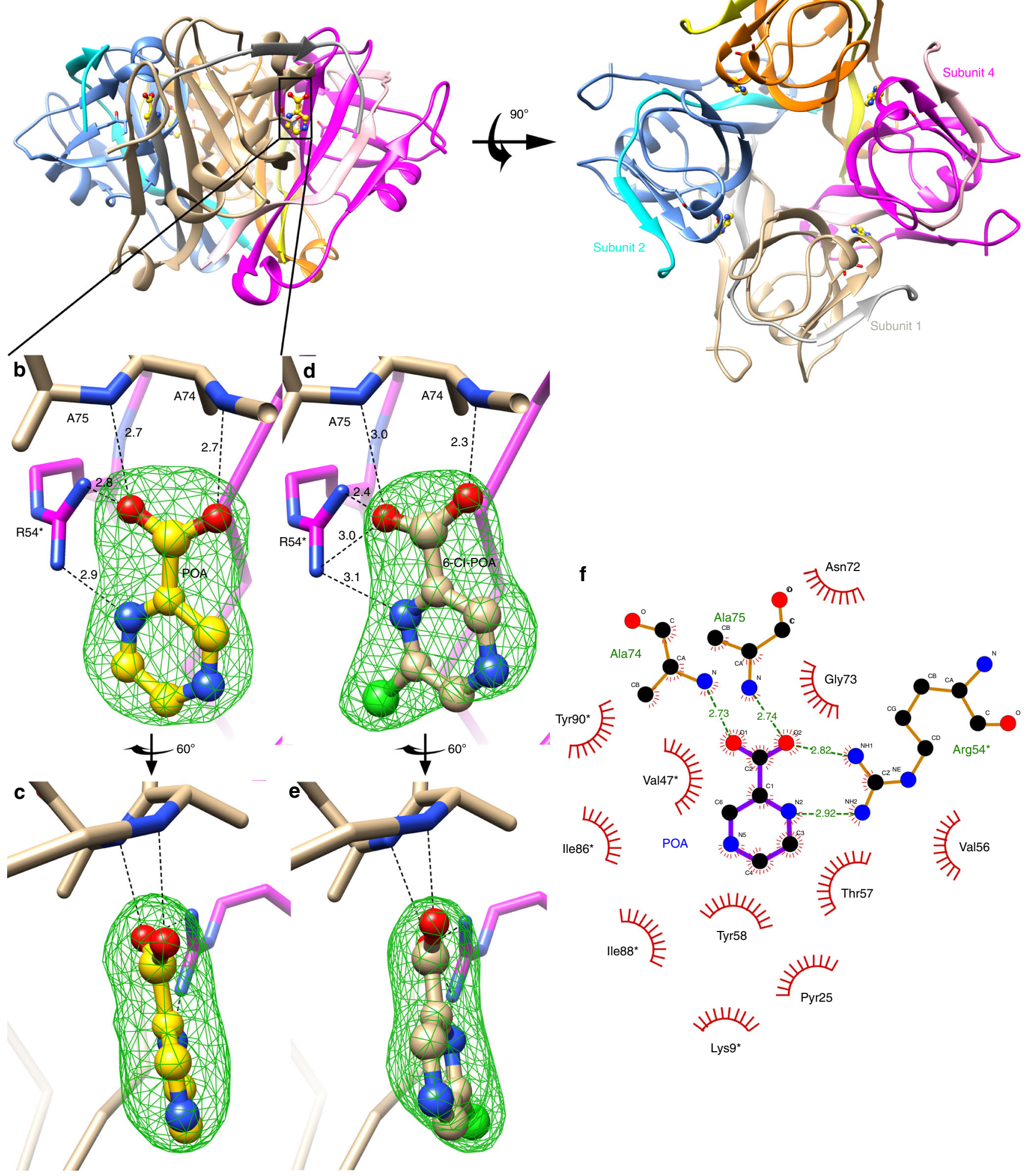

Fig. 2 Mtb PanD-POA interaction in the X-ray crystal structure. a POA binds Mtb PanD tetramer. The four subunits are shown in tan/silver, blue/cyan, orange/yellow, and magenta/pink. POA, shown in ball-and-stick, is bound to the active site at the interface of two PanD subunits. $\mathbf{b}$ Close up of the POA binding site. POA is shown with ball-and-stick with carbon in gold. The green mesh shows the polder omit map around POA at $3.5 \sigma$. Hydrogen bonds around POA are shown in dash lines. c The view is turned $60^{\circ}$ vertically. d, e 6-Cl-POA polder omit electron density. The PanD:6-Cl-POA is shown in the same view as PanD:POA in Fig. 2b, c. f POA shows extensive hydrogen bonding interactions with Mtb PanD. The interactions were analyzed and rendered with LigPlot ${ }^{+27}$. 
the surface by the two $C$-terminal loops of the $\alpha$ and $\beta$ chain. While we cannot be certain through which portal the substrate or inhibitor enters the active site, the protein must undergo a significant conformational change to allow substrate or POA access to the active site. This conformational change requirement for binding of molecules to the active site may account for the slow on- and off-rate of POA binding.

There were four hydrogen bonds that contribute significantly to the binding between POA and PanD: two between POAs carboxylate oxygens and the amide groups on the backbone of Ala74 $(2.7 \AA)$ and Ala75 $(2.7 \AA)$, one between a carboxylate oxygen to the guanidinium group of $\operatorname{Arg} 54^{*}(2.8 \AA)$, and another between the N1 on the POA pyrazine ring and Arg54* (2.9 $\AA$, Fig. 2f). When we mutated ${ }^{27}$ Arg54 to alanine, the inactive enzyme showed no binding to POA in the ITC experiment. This $\mathrm{H}$-bond explains the selectivity for the pyrazine ring and carboxylate consistent with the lack of inhibitory activity of both NA and PZA. Thus, POA binds to the active site of $M t b$ PanD interacting with the same groups as the substrate, and this is consistent with a competitive mode of inhibition.

PZA/POA resistance arises from mutations on active site loops. To investigate the mechanism of resistance, we purified recombinant protein from two of the most well-studied resistant mutants, H21R and M117 $\mathrm{I}^{11,19,20}$. Using ITC, we found that the $K_{\mathrm{d}}$ for POA binding to H21R was $2.84(0.06) \mathrm{mM}$ (Fig. 3a), and the $K_{\mathrm{i}}$ for POA inhibition of H21R was 3.0 (0.1) mM (Fig. 3b, c). The $K_{\mathrm{d}}$ for the mutant was $\sim 2.1 \mathrm{mM}$ higher than for the wildtype enzyme. However, resistance to POA came with a cost of enzyme activity: its $k_{\text {cat }}$ was $0.184(0.003) \mathrm{s}^{-1}$ compared to $0.330(0.006) \mathrm{s}^{-1}$ for the wild type, $K_{\mathrm{M}}$ was $5.5(0.2) \mathrm{mM}$ compared to $1.08(0.06) \mathrm{mM}$ for the wild type. We found that POA also bound to the M117I protein with significantly lower affinity compared to the wild type. The $K_{\mathrm{d}}$ was $\sim 200 \mu \mathrm{M}$ higher for the mutant than the wild type $\left(K_{\mathrm{d}}=0.90(0.09) \mathrm{mM}, n=4\right.$; Fig. 3a). The kinetics analysis showed that the POA off-rate measured by BLI $\left(k_{\text {off }}=0.051(0.008) \mathrm{s}^{-1}\right.$ and $0.06(0.01) \mathrm{s}^{-1}$ for the H21R and M117I mutants, respectively) was much faster than the wild-type $0.0027(0.0001) \mathrm{s}^{-1}$ (Fig. 3d). This suggests that the resistance of these two mutations is dependent on a combination of lower affinity as related to the decreased residence time of POA on PanD.

We crystallized and solved the structure of the M117I mutant protein. PanD M117I crystallized in the I422 space group and diffracted to $2.33 \AA$ resolution (Supplementary Table 1). The structure was very similar to the wild-type protein structure with a root-mean-square difference in the ca positions of $0.32 \AA$. The crystallization condition contained tartrate, a known inhibitor of the enzyme ${ }^{28}$. A molecule of tartrate fit well into electron density in the active site that could not be accounted for by the protein. The resulting structure showed reasonable interactions between tartrate and active site amino acids. In the mutant structure, we could fit an additional 11 residues at C-terminus that were not visible in electron density map for the wild-type protein. The ordered C-terminus enabled us to map several additional resistant mutation sites onto the structure, including M117, that were not visible in the wild-type structure (Fig. 3e). While none of the mutations were in amino acids that directly contacted POA, most were either on the $\mathrm{C}$-terminal loops of the $\alpha$ and $\beta$ chain or directly interacting with residues from these loops. As described above, these loops appear to form a flexible lid over the active site that sequesters the substrate from the solvent. That they are not directly in the active site is expected given the relatively small volume of the active site, where any mutation in the active site would likely be deleterious to the normal catalytic activity of the enzyme.

The biochemical and structural data serve to establish that PanD is the primary target of PZA. Our results show that resistance to PZA in PanD mutants primarily comes from alterations in two loops covering the active site that have subtle, but significant effects on the affinity and residence time of POA on the enzyme rather than from directly affecting POA binding interactions.

\section{Methods}

Protein expression and purification. Wild-type $M t b$ panD gene $(R v 3601 c)$ was PCR-amplified from $M t b$ genomic DNA and cloned into the pET21c $(+)$ vector ${ }^{29}$ All mutations were introduced through primers (Supplementary Table 2) using the DNA assembly kit (New England Biolabs). The plasmids were transformed into Escherichia coli BL21(DE3) cells for protein production. Protein expression was induced with $0.5 \mathrm{mM}$ IPTG at $\mathrm{OD}_{600} 0.6 \sim 1$, then the cells were incubated at $18^{\circ} \mathrm{C}$ overnight before harvesting by centrifugation. The cell pellets were resuspended in Ni-Buffer A (20 mM Tris, pH 8, $150 \mathrm{mM} \mathrm{NaCl}, 1 \mathrm{mM}$ 2-mercaptoethanol, and 5\% glycerol) with an additional $1 \mathrm{mM}$ PMSF and $1 \mathrm{mM}$ benzamidine, then mechanically lysed using a microfluidics with pressure setting at 20,000 psi. The lysate was cleared by centrifugation at $39,000 \times g$ for $1 \mathrm{~h}$ before application to HisTrap FF column (GE Healthcare). After extensive washing, the protein was eluted with linear gradient to $100 \%$ Ni-Buffer B (Ni-Buffer A + 500 $\mathrm{mM}$ imidazole). The fractions with PanD were pooled and dialyzed against $2 \mathrm{~L}$ Q-Buffer A (10 mM Tris, pH 8, $50 \mathrm{mM} \mathrm{NaCl}, 2 \mathrm{mM}$ DTT, and 5\% glycerol) before application to POROS HQ column (Thermofisher). Size-exclusion chromatography was carried out with a Sephacryl s-300 column (GE Healthcare) in buffer containing $20 \mathrm{mM}$ Tris, $\mathrm{pH} 8,50 \mathrm{mM} \mathrm{KCl}$, and $0.5 \mathrm{mM}$ TCEP. The final protein was concentrated to $\sim 100 \mathrm{mg} \mathrm{ml}^{-1}$, flash-frozen with liquid nitrogen, and kept at $-80^{\circ} \mathrm{C}$ freezer.

BLI. POA binding kinetics on $M t b$ PanD were characterized using an Octet RED96 System (fortéBIO, Pall Corp., USA). All reagents were buffered with $100 \mathrm{mM}$ Tris, pH 7.5. Biotinylated PanD were loaded onto eight High Precision Streptavidin (SAX) Biosensors (18-0037, fortéBIO, Pall Corp., USA), followed by a blocking step with biocytin. Then, a second baseline step was performed before samples (POA or PZA) at various concentrations were associated. The association and dissociation profiles were measured. Another set of eight biosensors were loaded with biocytin, processed in the same manner and served as the references (Supplementary Fig. 2a). Experiments were performed using the kinetics mode, at $28^{\circ} \mathrm{C}$ and sample plates were agitated at $1000 \mathrm{rpm}$. The data were analyzed with python/R script with the following routine: subtract eight references curves from the corresponding sample curves. Then the resulting curve with $0 \mathrm{mM}$ POA serving as the baseline was subtracted from the curves with POA ranging from $16 \mathrm{mM}$ to $0.25 \mathrm{mM}$. The baseline-corrected data were normalized with the second baseline step. The final association and dissociation curves were fitted with 'nls' function in R using Eq. (1):

$$
\operatorname{Response}\left(x_{\mathrm{i}}, t\right)=\left\{\begin{array}{l}
R_{\mathrm{a}}\left(x_{\mathrm{i}}, t\right)+a_{\mathrm{i}}+b_{\mathrm{i}} \times t, 0<t<\mathrm{T}_{\mathrm{a}} \\
R_{\mathrm{d}}\left(x_{\mathrm{i}}, t-\mathrm{T}_{\mathrm{a}}\right)+c_{\mathrm{i}}+d_{\mathrm{i}} \times\left(t-\mathrm{T}_{\mathrm{a}}\right), t>\mathrm{T}_{\mathrm{a}}
\end{array}\right.
$$

where

$$
\begin{gathered}
R_{\mathrm{a}}\left(x_{\mathrm{i}}, t\right)=\frac{R_{\mathrm{Max}} \times\left(1-e^{-\left(k_{\text {on }} \times x_{\mathrm{i}}+k_{\text {off }}\right) \times t}\right)}{1+\frac{k_{\text {off }}}{k_{\text {on }} \times x_{\mathrm{i}}}}, \\
R_{\mathrm{d}}\left(x_{\mathrm{i}}, t\right)=R_{\mathrm{a}}\left(x_{\mathrm{i}}, \mathrm{T}_{\mathrm{a}}\right) \times e^{-k_{\text {off }} \times\left(t-\mathrm{T}_{\mathrm{a}}\right)}
\end{gathered}
$$

Here, $x_{\mathrm{i}}$ is POA concentration; $\mathrm{T}_{\mathrm{a}}$ is the association period $(600 \mathrm{~s})$.

The $\mathrm{R}$ codes were built into an $\mathrm{R}$ package that we named 'smoke', and deposited in the CRAN repository [https://CRAN.R-project.org/package=smoke].

Enzymatic assays. The aspartate decarboxylase activity of PanD was coupled to the pantoate- $\beta$-alanine ligase (PanC) reaction. The generation of pyrophosphate from this reaction was detected with the EnzChek ${ }^{\mathrm{TM}}$ Pyrophosphate Assay Kit (ThermoFisher E6645). The reaction volume was $100 \mu \mathrm{l}$. In each reaction, there is $0.2 \mu \mathrm{M} M t b \mathrm{PanD}, 3 \mu \mathrm{M} M t b$ PanC, $2 \mathrm{mM}$ ATP, $2 \mathrm{mM}$ pantoate, $100 \mathrm{mM}$ HEPES ( $\mathrm{pH} 7$ ), and $10 \mathrm{mM} \mathrm{MgCl}_{2}$. All reactions were carried out at $28^{\circ} \mathrm{C}$ in a CLAIROstar plate reader (BMG Labtech) and the absorbance at $360 \mathrm{~nm}$ was measured. The data analysis and model fitting were done with R scripting [http://www.R-project.org].

ITC. ITC experiments were performed using a MicroCal iTC200 (GE). Both protein and ligands were in a buffer of $100 \mathrm{mM}$ Tris, $\mathrm{pH}$ 7.5. The protein concentration in the cell was $0.5 \mathrm{mM}$, and the ligand concentration was $20 \mathrm{mM}$. The titration was performed at $20^{\circ} \mathrm{C}$. The data were analyzed with the Origin 7.0 software and fitted with a single-site binding model. 
a

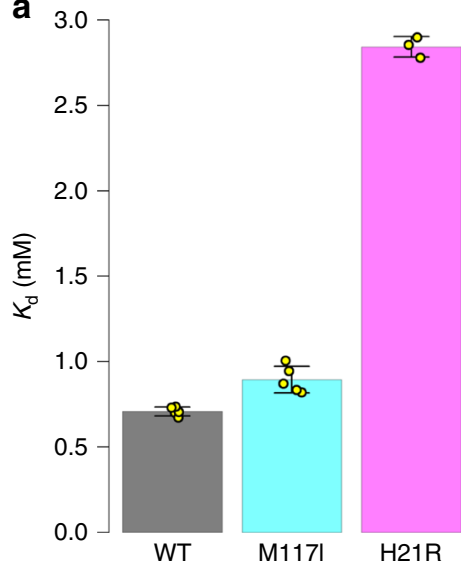

b

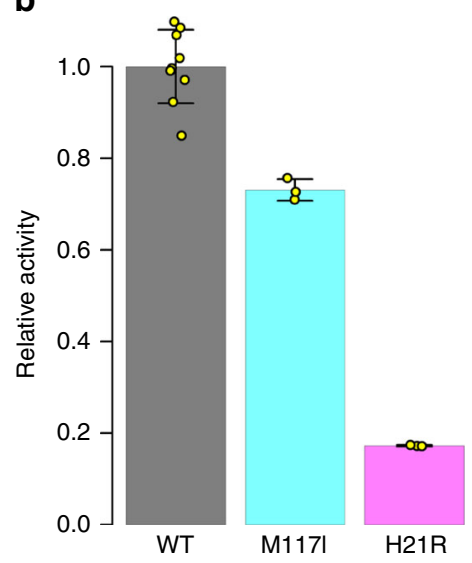

C

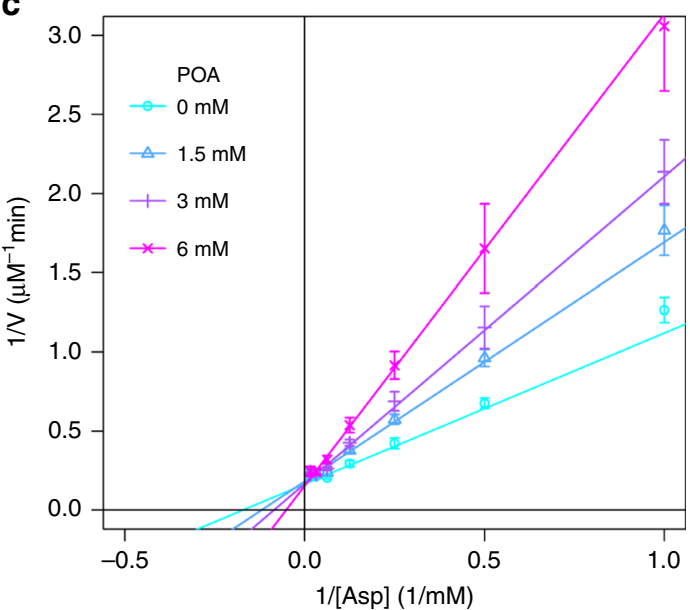

e

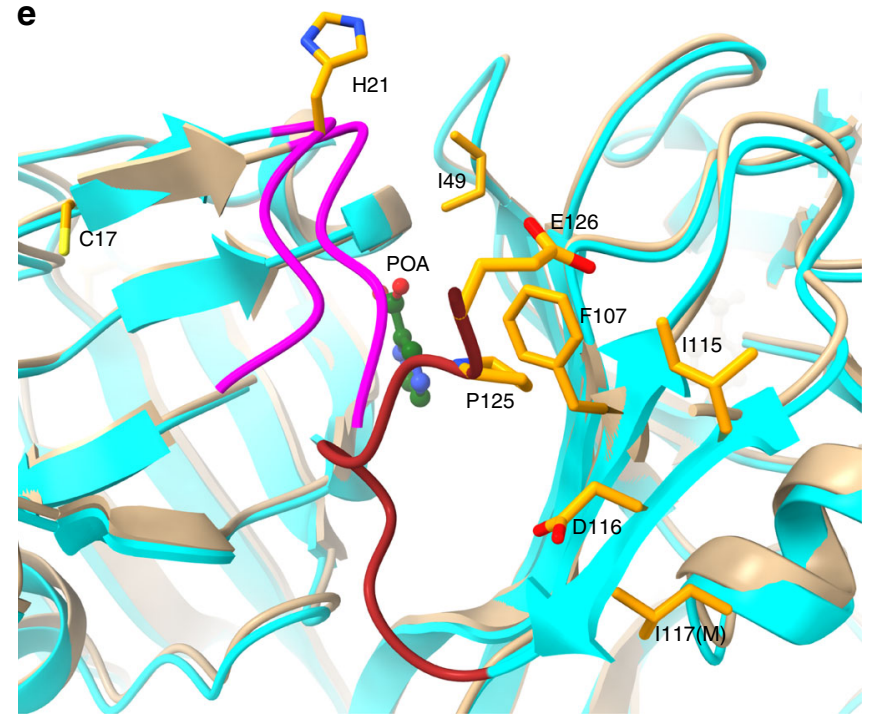

d

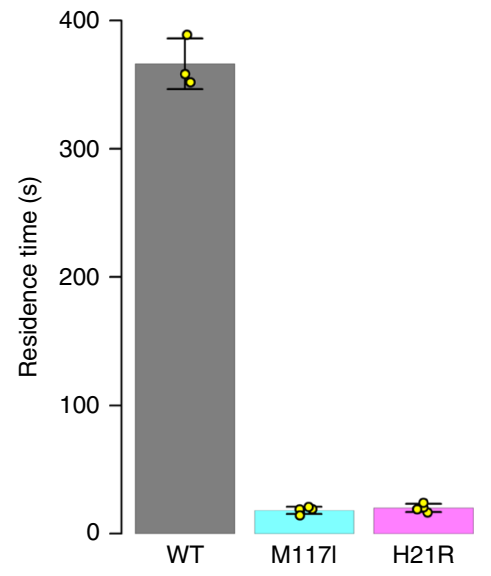

Fig. 3 POAr mutations on Mtb PanD. a H21R showed resistance to POA. Lineweaver-Burk plots of H21R activity in the presence of various concentrations of POA prepared separately in quadruplicate. The data were fitted with a competitive inhibition model, yielding $K_{\mathrm{i}}=3.0(0.1) \mathrm{mM}, K_{\mathrm{M}}=5.5(0.2) \mathrm{mM}$, and $k_{\text {cat }}=0.184(0.003) \mathrm{s}^{-1}$. b The relative enzyme activity of $M t b$ PanD proteins. The aspartate concentration was $1 \mathrm{mM}$ in the assay. c The affinity of $M t b$ PanD:POA was measured with ITC. POA at $40 \mathrm{mM}$ was titrated into $0.5 \mathrm{mM} \mathrm{H} 21 \mathrm{R}$ PanD, while $20 \mathrm{mM}$ POA was used in the wild type and M117I experiments. d Residence time of POA-panD measured with BLI. e Mapping of POAr sites on M117l and wild-type PanD:POA structures. A ribbon structure of the M117I mutant colored in cyan is superimposed on to the wild-type PanD ribbon in tan. Side chains of the reported resistant mutants mapped to the M117I structure are shown in orange. POA is shown in green. The $\beta$-chain C-terminal loop Leu20-Gly24 is highlighted in magenta, while the $\alpha$-chain loop His119-Glu126 is in brown. Error bars were defined as standard deviations. Source data are provided as a Source Data file.

Crystallization and data collection. Protein stocks stored in $-80^{\circ} \mathrm{C}$ were quickly thawed and diluted to $20 \mathrm{mg} \mathrm{ml}^{-1}$ for all crystallization trials. Hanging-drop vapor method was used at $16^{\circ} \mathrm{C}$ to produce PanD crystals. For 6-Cl-POA complex crystals, $20 \mathrm{mM}$ ligand was premixed in the protein solution. Wild-type Mtb PanD crystals were produced in mother liquor containing $15-18 \%$ PEG 3350, $200 \mathrm{mM}$ ammonium chloride, and $100 \mathrm{mM}$ MES/HEPES pH 6.5-7. The cryo-protectant containing $20 \%$ PEG 3350, $200 \mathrm{mM}$ ammonium chloride, $100 \mathrm{mM}$ HEPES pH 7, and 15\% glycerol. A total of $10 \mathrm{mM}$ POA or 6-Cl-POA was mixed in the cryo-protectant for the respective complex crystals. The crystals were immediately flash cooled in liquid nitrogen. The diffraction data were collected at APS 23ID and processed with Proteum 3 software (Bruker). PanD M117I crystallized at $20 \mathrm{mg} \mathrm{ml}^{-1}$ in mother liquor containing $100 \mathrm{mM}$ ammonium tartrate and $12 \%$ PEG3350. Additional $15 \%$ glycerol was added into the mother liquor as a cryo-protectant to flash cool crystals. The diffraction data were collected at APS 19ID and processed with HKL3000 ${ }^{30}$

Structural determination and refinement. The initial Apo Mtb PanD model was from the molecular replacement with pdb ID: 2C45 as the search template in phaser ${ }^{31}$. This model was used as the template in molecular replacement for all the other PanD structures in this study. Structure refinement was performed in Refmac5 and Phenix refine, combined with manual correction and rebuild iteratively. The final structural model of PanD:POA complex was refined to Rwork Rfree of $0.1992 / 0.2478$. In the final Ramachandran plot, $99.55 \%$ and $0.45 \%$ of residues were in the favored and allowed regions, respectively. The data collection and refinement statistics are listed in Supplementary Table 1. All structure figures were drawn in Chimera.

Reporting summary. Further information on research design is available in the Nature Research Reporting Summary linked to this article.

\section{Data availability}

Coordinates and structure factors have been deposited in the Protein Data Bank under accession codes: 6OZ8 for Apo_PanD, 6OYY for POA:PanD, 6P02 for 6-Cl-POA: PanD, and 6P1Y for M117I mutant. Figure data can be found at [https://github.com/ quinsun/mtbPanD]. Other data are available from the corresponding author upon request.

\section{Code availability}

Code used for analyzing BLI kinetics and the related dataset has been built into an $\mathrm{R}$ package, we named 'smoke', and deposited at the CRAN repository [https://CRAN.Rproject.org/package $=$ smoke]. 
Received: 5 September 2019; Accepted: 16 December 2019;

Published online: 17 January 2020

\section{References}

1. WHO. Global tuberculosis report 2018. Glob. Tuberc. Rep. 2018, 1-231 https://reliefweb.int/report/world/global-tuberculosis-report-2018 (2018).

2. Zhang, Y., Shi, W., Zhang, W. \& Mitchison, D. Mechanisms of pyrazinamide action and resistance. Microbiol Spectr. 2, 1-12 (2013).

3. WHO Treatment Guidelines for Drug-Resistant Tuberculosis, 2016 Update https://apps.who.int/iris/bitstream/handle/10665/250125/9789241549639-eng. pdf (Geneva, 2016)

4. Yeager, R. L., Munroe, W. G. \& Dessau, F. I. Pyrazinamide (aldinamide) in the treatment of pulmonary tuberculosis. Am. Rev. Tuberc. 65, 523-546 (1952).

5. Tarshis, M. S. \& Weed, W. A. Jr. Lack of significant in vitro sensitivity of Mycobacterium tuberculosis to pyrazinamide on three different solid media. Am. Rev. Tuberc. 67, 391-395 (1953).

6. Zhang, Y., Permar, S. \& Sun, Z. Conditions that may affect the results of susceptibility testing of Mycobacterium tuberculosis to pyrazinamide. J. Med. Microbiol. 51, 42-49 (2002).

7. Salfinger, M. \& Heifets, L. B. Determination of pyrazinamide MICs for Mycobacterium tuberculosis at different $\mathrm{pHs}$ by the radiometric method. Antimicrob. Agents Chemother. 32, 1002-1004 (1988).

8. Scorpio, A. \& Zhang, Y. Mutations in pncA, a gene encoding pyrazinamidase/ nicotinamidase, cause resistance to the antituberculous drug pyrazinamide in tubercle bacillus. Nat. Med. 2, 662-667 (1996).

9. Shi, W. et al. Introducing RpsA point mutations $\triangle 438 \mathrm{~A}$ and D123A into the chromosome of M. tuberculosis confirms their role in causing resistance to pyrazinamide. Antimicrob. Agents Chemother. 63, e02681-18 (2019).

10. Shi, W., Chen, J., Zhang, S., Zhang, W. \& Zhang, Y. Identification of novel mutations in Lprg (rv1411c), rv0521, rv3630, rv0010c, ppsc, and cyp128 associated with pyrazinoic acid/pyrazinamide resistance in Mycobacterium tuberculosis. Antimicrob. Agents Chemother. 62 (2018).

11. Gopal, P. et al. In vivo-selected pyrazinoic acid-resistant Mycobacterium tuberculosis strains harbor missense mutations in the aspartate decarboxylase PanD and the unfoldase ClpC1. ACS Infect. Dis. 3, 492-501 (2017).

12. Dillon, N. A., Peterson, N. D., Feaga, H. A., Keiler, K. C. \& Baughn, A. D. Anti-tubercular activity of pyrazinamide is independent of trans-translation and RpsA. Sci. Rep. 7, 6135 (2017).

13. Peterson, N. D., Rosen, B. C., Dillon, N. A. \& Baughn, A. D. Uncoupling environmental $\mathrm{ph}$ and intrabacterial acidification from pyrazinamide susceptibility in Mycobacterium tuberculosis. Antimicrob. Agents Chemother. 59, 7320-7326 (2015).

14. Dillon, N. A., Peterson, N. D., Rosen, B. C. \& Baughn, A. D. Pantothenate and pantetheine antagonize the antitubercular activity of pyrazinamide. Antimicrob. Agents Chemother. 58, 7258-7263 (2014).

15. Shi, W. et al. Pyrazinamide inhibits trans-translation in Mycobacterium tuberculosis. Science 333, 1630-1632 (2011).

16. Zhang, Y., Wade, M. M., Scorpio, A., Zhang, H. \& Sun, Z. Mode of action of pyrazinamide: disruption of Mycobacterium tuberculosis membrane transport and energetics by pyrazinoic acid. J. Antimicrob. Chemother. 52, 790-795 (2003).

17. Boshoff, H. I., Mizrahi, V. \& Barry, C. E. 3rd Effects of pyrazinamide on fatty acid synthesis by whole mycobacterial cells and purified fatty acid synthase I. J. Bacteriol. 184, 2167-2172 (2002).

18. Zimhony, O., Cox, J. S., Welch, J. T., Vilcheze, C. \& Jacobs, W. R. Jr. Pyrazinamide inhibits the eukaryotic-like fatty acid synthetase I (FASI) of Mycobacterium tuberculosis. Nat. Med. 6, 1043-1047 (2000).

19. Shi, W. et al. Aspartate decarboxylase (PanD) as a new target of pyrazinamide in Mycobacterium tuberculosis. Emerg. Microbes Infect. 3, e58 (2014).

20. Zhang, S. et al. Mutations in panD encoding aspartate decarboxylase are associated with pyrazinamide resistance in Mycobacterium tuberculosis Emerg. Microbes Infect. 2, e34 (2013).

21. Sambandamurthy, V. K. et al. A pantothenate auxotroph of Mycobacterium tuberculosis is highly attenuated and protects mice against tuberculosis. Nat. Med. 8, 1171-1174 (2002).

22. Gopal, P. et al. Pyrazinoic acid inhibits mycobacterial coenzyme A biosynthesis by binding to aspartate decarboxylase PanD. ACS Infect. Dis. 3, 807-819 (2017).

23. Concepcion, J. et al. Label-free detection of biomolecular interactions using BioLayer interferometry for kinetic characterization. Comb. Chem. High. Throughput Screen 12, 791-800 (2009).

24. Liebschner, D. et al. Polder maps: improving OMIT maps by excluding bulk solvent. Acta Crystallogr. D Struct. Biol. 73, 148-157 (2017).
25. Lee, B. I. \& Suh, S. W. Crystal structure of the schiff base intermediate prior to decarboxylation in the catalytic cycle of aspartate alpha-decarboxylase. J. Mol. Biol. 340, 1-7 (2004).

26. Friesner, R. A. et al. Extra precision glide: docking and scoring incorporating a model of hydrophobic enclosure for protein-ligand complexes. J. Med. Chem. 49, 6177-6196 (2006).

27. Laskowski, R. A. \& Swindells, M. B. LigPlot+: multiple ligand-protein interaction diagrams for drug discovery. J. Chem. Inf. Model 51, 2778-2786 (2011).

28. Sharma, R., Florea, M., Nau, W. M. \& Swaminathan, K. Validation of druglike inhibitors against Mycobacterium tuberculosis L-aspartate alphadecarboxylase using nuclear magnetic resonance (1H NMR). PLoS ONE 7, e45947 (2012).

29. Chopra, S., Pai, H. \& Ranganathan, A. Expression, purification, and biochemical characterization of Mycobacterium tuberculosis aspartate decarboxylase, PanD. Protein Expr. Purif. 25, 533-540 (2002).

30. Minor, W., Cymborowski, M., Otwinowski, Z. \& Chruszcz, M. HKL-3000: the integration of data reduction and structure solution-from diffraction images to an initial model in minutes. Acta Crystallogr. D Biol. Crystallogr. 62, 859-866 (2006).

31. McCoy, A. J. et al. Phaser crystallographic software. J. Appl. Crystallogr. 40, 658-674 (2007).

\section{Acknowledgements}

Funding for this work came from the TB Structural Genomics Consortium, P01AI095208 from NIAID, NIH (J.C.S.), grant A-0015 from the Welch Foundation (J.C.S.), and a grant from the Chancellor Research Initiative-Texas A\&M System (J.C.S.). Results shown in this report are derived from work performed at Argonne National Laboratory, a U.S. Department of Energy (DOE) Office of Science User Facility operated for the DOE Office of Science by Argonne National Laboratory. Data were collected from the Structural Biology Center (SBC) at the Advanced Photon Source (Argonne National Laboratory). SBC-CAT is operated by U. Chicago Argonne, LLC, for the U.S. Department of Energy, Office of Biological and Environmental Research under contract DE-AC02-06CH11357. We thank the staff at beamline 19-ID and 23-ID for assistance with data collection. We thank Dr. Steve Lockless for advice on ITC and reading the manuscript, Dr. Matthews Benning for advice on X-ray data processing with Proteum (Bruker), and Tracey Musa for editing the manuscript.

\section{Author contributions}

J.C.S. and Q.S. designed the experiments and wrote the manuscript; Q.S. and X.L. performed the experiments and the data analysis; L.M.P. instructed the ligand docking; and Y.Z. and W.S. contributed to the writing.

\section{Competing interests}

The authors declare no competing interests.

\section{Additional information}

Supplementary information is available for this paper at https://doi.org/10.1038/s41467 019-14238-3.

Correspondence and requests for materials should be addressed to J.C.S.

Peer review information Nature Communications thanks the anonymous reviewer(s) for their contribution to the peer review of this work. Peer reviewer reports are available.

Reprints and permission information is available at http://www.nature.com/reprints

Publisher's note Springer Nature remains neutral with regard to jurisdictional claims in published maps and institutional affiliations.

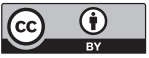

Open Access This article is licensed under a Creative Commons Attribution 4.0 International License, which permits use, sharing, adaptation, distribution and reproduction in any medium or format, as long as you give appropriate credit to the original author(s) and the source, provide a link to the Creative Commons license, and indicate if changes were made. The images or other third party material in this article are included in the article's Creative Commons license, unless indicated otherwise in a credit line to the material. If material is not included in the article's Creative Commons license and your intended use is not permitted by statutory regulation or exceeds the permitted use, you will need to obtain permission directly from the copyright holder. To view a copy of this license, visit http://creativecommons.org/ licenses/by/4.0/

(c) The Author(s) 2020 